\title{
ON THE ZEROS OF THE DIRICHLET $L$-FUNCTIONS NEAR THE CRITICAL LINE
}

TOM MEURMAN

\section{Introduction.}

Let $\chi$ be a Dirichlet character $\bmod q$ and $\chi_{0}$ the principal character $\bmod q$. Denote by $N_{\chi}\left(\alpha ; T_{1}, T_{2}\right)$ the number of zeros of the Dirichlet $L$-function $L(s, \chi)$ in the rectangle $\alpha \leqq \sigma \leqq 1, T_{1} \leqq t \leqq T_{2}$. Write briefly $N_{\chi}(\alpha ; T)=N_{\chi}(\alpha ;-T, T)$ and $N(\alpha ; T)=N_{\chi_{0}}(\alpha ; T)$. We shall give for the number

$$
N(q)=\sum_{\chi \bmod q} N_{\chi}\left(\alpha ; T_{1}, T_{2}\right)
$$

an upper bound which is interesting when $\alpha$ is near $\frac{1}{2}$. Results of this type were first obtained by Selberg. In [7] he proved namely that

$$
N(\alpha ; T) \ll T^{1-\left(\alpha-\frac{1}{2}\right) / 4} \log T
$$

for $\frac{1}{2} \leqq \alpha \leqq 1$ and $T \geqq 2$. In [8] he proved that if $\left|T_{1}\right|,\left|T_{2}\right| \leqq q^{\frac{1}{4}-\varepsilon}, T_{2}-T_{1} \geqq 1 / \log q$ and $\frac{1}{2}+1 / \log q \leqq \alpha<1$, then

$$
N^{\prime}(q) \ll q^{1-\frac{2}{3}\left(\alpha-\frac{1}{2}\right) \varepsilon}\left(T_{2}-T_{1}\right) \log q,
$$

where ' means that $\chi_{0}$ is omitted in the summation. Recently Jutila [3] proved that for $\frac{1}{2} \leqq \alpha \leqq 1, T \geqq 2$ and any fixed $\varepsilon>0$,

$$
N(\alpha ; T) \ll_{\varepsilon} T^{1-(1-\varepsilon)\left(\alpha-\frac{1}{2}\right)} \log T,
$$

which is a sharpened version of (1.2). We combine methods of Selberg [8], Jutila [3] and Ramachandra [6] to prove the following theorem.

THEOREM. Let $q$ be a positive integer, $\alpha \geqq \frac{1}{2}, T_{2}-T_{1} \gg 1 / \log (q+1), 0<2 \varepsilon \leqq c$ $\leqq 1$ and

$$
\max \left(\left|T_{1}\right|,\left|T_{2}\right|\right) \ll q^{1-c}\left(T_{2}-T_{1}+1\right) .
$$

Then, in the notation (1.1), we have

$$
\begin{aligned}
N(q) & \ll_{\varepsilon} q^{1-(c-\varepsilon)\left(\alpha-\frac{1}{2}\right)}\left(T_{2}-T_{1}\right) \times \\
& \times\left(T_{2}-T_{1}+1\right)^{-(1-\varepsilon)\left(\alpha-\frac{1}{2}\right)} \log q\left(T_{2}-T_{1}+2\right) .
\end{aligned}
$$

Received January 8, 1982. 
Corollary 1. If $T \geqq 2$, then

$$
\sum_{\chi \bmod q} N_{\chi}(\alpha ; T) \ll_{\varepsilon}(q T)^{1-(1-\varepsilon)\left(\alpha-\frac{1}{2}\right)} \log q T .
$$

This is a generalization of $(1.4)$ and for $\frac{1}{2} \leqq \alpha \leqq \frac{1}{2}+(24-\varepsilon)(\log \log q T) / \log q T$ it is sharper than the estimate of Montgomery ([4, Theorem 12.1]), which is a generalization of Ingham's [2] well-known theorem.

Corollary 2. If $T_{2}-T_{1} \gg 1 / \log (q+1)$ and

$$
\max \left(\left|T_{1}\right|,\left|T_{2}\right|\right) \ll q^{1-2 \varepsilon},
$$

then

$$
N(q) \ll_{\varepsilon}\left(T_{2}-T_{1}\right) q^{1-\varepsilon\left(\alpha-\frac{1}{2}\right)} \log (q+1) .
$$

This is both sharper and more general than (1.3). In particular it enables one to estimate nontrivially the number of zeros in a rectangle of height $1 / \log q$ at a distance $q^{1-\varepsilon}$ (instead of $q^{\frac{1}{4}-\varepsilon}$ ) from the real axis.

Both of the corollaries are immediate consequences of the theorem.

Now we shall outline the proof and introduce some notation. Let

$$
\begin{gathered}
1<z_{1}<z_{2}, \\
1<v_{1}<v_{2}, \\
x_{n}=x_{n}\left(v_{1}, v_{2}\right)= \begin{cases}1, & 1 \leqq n \leqq v_{1}, \\
\log \left(v_{2} / n\right) / \log \left(v_{2} / v_{1}\right), & v_{1}<n \leqq v_{2}, \\
0, & n>v_{2},\end{cases} \\
\lambda_{n}=\lambda_{n}\left(z_{1}, z_{2}\right)=\mu(n) x_{n}\left(z_{1}, z_{2}\right),
\end{gathered}
$$

where $\mu(n)$ is the Möbius function. Then the "mollifier"

$$
M(s, \chi)=\sum_{n<z_{2}} \chi(n) \lambda_{n} n^{-s}
$$

makes the quantity $|M(s, \chi) L(s, \chi)-1|^{2}$ small on the average, but at the zeros of $L(s, \chi)$ this expression equals 1 : this argument gives the density estimate. It turns out, as was observed by Jutila [3] in the case $\chi=\chi_{0}$, that the mean value of the same expression with $L(s, \chi)$ replaced by the smoothened partial sum

$$
F(s, \chi)=\sum_{n<v_{2}} \chi(n) x_{n} n^{-s}
$$

can be estimated satisfactorily. In order to make use of this fact in estimating the mean value of $|M(s, \chi) L(s, \chi)-1|^{2}$, we appeal to an idea of Ramachandra 
[6] (see Lemma 2 below). The problem then reduces to estimating sums of the type $\sum a_{n}^{2} n^{-2 \sigma}$ and $\sum b_{n}^{2}(x) n^{-1}$, where

$$
a_{n}=a_{n}\left(z_{1}, z_{2}\right)=\sum_{d \mid n} \lambda_{d}
$$

and

$$
b_{n}(x)=b_{n}\left(x ; v_{1}, v_{2}, z_{1}, z_{2}\right)=\sum_{d \mid n} \lambda_{d} x_{n / d} d^{x}
$$

in particular the numbers $b_{n}(0)$ are the coefficients of the Dirichlet polynomial $M(s, \chi) F(s, \chi)$. The estimates of these sums, given in Lemmas 4 and 5 , are based on Lemma 3, which is in some respects more general than the corresponding lemma of Motohashi [5].

2. A formula for $M(s, \chi) L(s, \chi)$.

LEMMA 1. Let

$$
L(s, \chi)=\Psi(s, \chi) L(1-s, \bar{\chi}) .
$$

Then $\Psi(s, \chi)$ is holomorphic in the region $\sigma<1$. If $A \leqq \sigma \leqq \frac{1}{2}$, then

$$
\Psi(s, \chi) \ll_{A}(q(|t|+1))^{\frac{1}{2}-\sigma},
$$

whether $\chi$ is primitive or not.

The proof of lemma 1 is well-known.

Lemma 2. If $X>1,0<y<\frac{1}{4}, \sigma \geqq \frac{1}{2}-y, h>2 \sigma$ and $\chi \neq \chi_{0}$, then

$$
M(s, \chi) L(s, \chi)=\exp \left(-X^{-h}\right)+S(s, X, \chi)-I_{0}(s, X, \chi)-I_{1}(s, X, \chi),
$$

where

$$
\begin{gathered}
I_{j}(s, X, \chi)=\frac{1}{2 \pi i} \int_{C,} \Psi(s+w, \chi) M(s+w, \chi) \times \\
\times(j L(1-s-w, \bar{\chi})+(1-2 j) F(1-s-w, \bar{\chi})) \Gamma\left(1+\frac{w}{h}\right) X^{w} w^{-1} d w ; j=0,1,
\end{gathered}
$$$$
S(s, X, \chi)=\sum_{n>z_{1}} \chi(n) a_{n} \exp \left(-(n / X)^{h}\right) n^{-s},
$$

and the paths $C_{j}$ are defined as follows: 


$$
\begin{aligned}
C_{0}= & \left\{w:|\operatorname{Im} w|<y, \operatorname{Re} w=\min \left(-y, \frac{1}{2}-\sigma\right)\right\} \\
& \cup\left\{w:|\operatorname{Im} w|=y, \min \left(-y, \frac{1}{2}-\sigma\right) \leqq \operatorname{Re} w \leqq \frac{1}{2}-\sigma\right\} \\
& \cup\left\{w:|\operatorname{Im} w|>y, \operatorname{Re} w=\frac{1}{2}-\sigma\right\}, \\
C_{1}= & \{w: \operatorname{Re} w=-h / 2\} .
\end{aligned}
$$

Proof. By Mellin's transformation, we have

$$
\begin{aligned}
& \sum_{n=1}^{\infty} \chi(n) a_{n} \exp \left(-(n / X)^{h}\right) n^{-s} \\
&=\frac{1}{2 \pi i} \int_{\operatorname{Re} w=2} M(s+w, \chi) L(s+w, \chi) \Gamma\left(1+\frac{w}{h}\right) X^{w} w^{-1} d w .
\end{aligned}
$$

We shift the line of integration to the line $C_{1}$. The pole at $w=0$ gives then the term $M(s, \chi) L(s, \chi)$. By $(2.1)$

$$
L(s+w, \chi)=\Psi(s+w, \chi) \sum_{j=0}^{1}(j L(1-s-w, \bar{\chi})+(1-2 j) F(1-s-w, \bar{\chi})) .
$$

Thus the integral over $C_{1}$ equals $I_{0}(s, X, \chi)+I_{1}(s, X, \chi)$, since the integrand of $I_{0}(s, X, \chi)$ has no singularities between $C_{0}$ and $C_{1}$.

\section{A lemma related to Selberg's sieve.}

Let $\sigma_{a}(n)$ denote the sum of the $a$ th powers of the divisors of $n$. It is easy to see that if $z>1, a \geqq 1, b \gg 1$, and $c \ll 1$, then

$$
\sum_{m<z} m^{-a} \sigma_{-b}^{c}(m) \ll \log z .
$$

The following lemma is a modification of a lemma of Motohashi [5].

Lemma 3. For $z>1$, let

$$
\left\{\begin{array}{l}
1 \ll(\omega-1) \log z \ll 1 \\
1 \ll\left(\omega^{\prime}-1\right) \log z \ll 1
\end{array}\right.
$$

and define

$$
D_{r}=\sum_{n=1}^{\infty}\left(\sum_{\substack{d \mid n \\ d<z}} \mu\left(d^{r}(n / d)^{1-r}\right) d^{\omega-\omega^{\prime}} \log ^{k}(z / d)\right)^{2} n^{-\omega}
$$

Then

$$
D_{r} \ll_{k}(\log z)^{2 k},
$$

for any positive integer $k$ and for $r=0$ or $r=1$. 
Proof. We have

$$
\begin{gathered}
D_{r}=\sum_{d_{1}, d_{2}<z}\left[d_{1}, d_{2}\right]^{-\omega}\left(\prod_{j=1}^{2} d_{j}^{\omega-\omega^{\prime}} \mu\left(d_{j}\left(d_{1}, d_{2}\right)^{r-1}\right) \log ^{k}\left(z / d_{j}\right)\right) \times \\
\times \sum_{\substack{n=1 \\
\left(n^{1-r}, d_{1} d_{2}\left(d_{1}, d_{2}\right)^{-2}\right)=1}}^{\infty} \mu^{2}\left(n^{1-r}\right) n^{-\omega} .
\end{gathered}
$$

Here the series over $n$ equals

$$
\zeta(\omega)\left(\zeta(2 \omega) \prod_{j=1}^{2} \sigma_{-\omega}\left(d_{j} /\left(d_{1}, d_{2}\right)\right)\right)^{r-1},
$$

since we may assume that $d_{j} /\left(d_{1}, d_{2}\right)$ is square-free. Writing $\left(d_{1}, d_{2}\right)=d, d_{1}=d e_{1}$, $d_{2}=d e_{2}$ and summing first with respect to $e_{1}$ and $e_{2}$, we have further

$$
D_{r}=\zeta(\omega)(\zeta(2 \omega))^{r-1} E_{r},
$$

where

$$
E_{r}=\sum_{d<z} \mu^{2}\left(d^{r}\right) d^{\omega-2 \omega^{\prime}} \sum_{\begin{array}{c}
e_{1}, e_{2}<z / d \\
\left(e_{2}, e_{2}\right)=1 \\
\left(e_{1}, d^{\prime}\right)=\left(e_{2}, d^{\prime}\right)=1
\end{array}} \prod_{j=1}^{2} F_{e_{,}, r}(z / d)
$$

and

$$
F_{u, r}(x)=u^{-\omega^{\prime}} \mu(u) \log ^{k}(x / u) \sigma_{-\omega}^{r-1}(u) .
$$

The inner double sum in the expression for $E_{r}$ is rewritten as

$$
\sum_{\substack{a<z / d \\\left(a, d^{\prime}\right)=1}} \mu(a)\left(\sum_{\substack{u<z / a d \\\left(u, d^{\prime}\right)=1}} F_{a u, r}(z / d)\right)^{2} .
$$

Hence, writing $a d=m$, we have

$$
E_{r}=\sum_{m<z} m^{\omega-2 \omega^{\prime}} \mu^{2}\left(m^{r}\right) \sum_{a \mid m} \mu(a) a^{-\omega} \sigma_{-\omega}^{2 r-2}(a)\left(R_{a^{1-r} m^{r}, r}(z / m)\right)^{2},
$$

where

$$
R_{v, r}(x)=\sum_{\substack{u<x \\(u, v)=1}} F_{u, r}(x)
$$

Let $\chi_{0}^{(v)}$ be the principal character $\bmod v$. The generating function of the arithmetic function $u^{-\omega^{\prime}} \mu(u) \sigma_{-\omega}^{r-1}(u) \chi_{0}^{(v)}(u)$ is $P_{v, r}\left(s+\omega^{\prime}\right)$, where 


$$
\begin{aligned}
P_{v, r}(s) & =(\zeta(s))^{-1}(G(s))^{1-r} H_{v, r}(s), \\
G(s) & =\prod_{p}\left(1+\left(\left(p^{s}-1\right)\left(p^{\omega}+1\right)\right)^{-1}\right), \\
H_{v, r}(s) & =\prod_{p \mid v}\left(1-p^{-s}\left(1+p^{-\omega}\right)^{r-1}\right)^{-1} .
\end{aligned}
$$

Indeed,

$$
\sum_{u=1}^{\infty} u^{-s} \mu(u) \sigma_{-\omega}^{r-1}(u) \chi_{0}^{(v)}(u)=K_{r}(s) H_{v, r}(s)
$$

where

$$
K_{r}(s)=\prod_{p}\left(1-p^{-s}\left(1+p^{-\omega}\right)^{r-1}\right)
$$

whence

$$
\begin{aligned}
K_{0}(s) & =\prod_{p}\left(1-p^{-s}\right)\left(1+\frac{p^{-s}\left(1-\left(1+p^{-\omega}\right)^{-1}\right)}{1-p^{-s}}\right) \\
& =(\zeta(s))^{-1} G(s)
\end{aligned}
$$

and

$$
K_{1}(s)=(\zeta(s))^{-1}
$$

Hence we have the representation of $R_{v, r}(x)$ as an integral

$$
R_{v, r}(x)=\frac{k !}{2 \pi i} \int_{2-i \infty}^{2+i \infty} P_{v, r}\left(s+\omega^{\prime}\right) x^{s} s^{-k-1} d s .
$$

We move the integration to the path $C$ defined by

$$
C=\left\{s=\sigma+i t: \sigma=1-\omega^{\prime}-\Delta / \log (|t|+2)\right\},
$$

where $\Delta$ is a small positive constant. This shows that

$$
R_{v, r}(x)=k ! \operatorname{Res}\left(P_{v, r}\left(s+\omega^{\prime}\right) x^{s} s^{-k-1}\right)_{s=0}+O_{k}\left(\sigma_{-\frac{1}{2}}(v)\right),
$$

since, for $s \in C$, we have

$$
\begin{gathered}
\zeta\left(s+\omega^{\prime}\right)^{-1} \ll \log (|t|+2), \\
G\left(s+\omega^{\prime}\right) \ll 1, \\
H_{v, r}\left(s+\omega^{\prime}\right) \ll \sigma_{-\frac{1}{2}}(v) .
\end{gathered}
$$

The first of these three estimates and the fact that the origin is the only 
singularity of the integrand on the right hand side of $C$ are well-known classical results.

To calculate the residue, we note that near $s=0$

$$
\begin{gathered}
\zeta\left(s+\omega^{\prime}\right)^{-1}=O\left(\omega^{\prime}-1\right)+\sum_{j=1}^{\infty} O_{j}(1) s^{j}, \\
G\left(s+\omega^{\prime}\right)=\sum_{j=0}^{\infty} O_{j}(1) s^{j}, \\
H_{v, r}\left(s+\omega^{\prime}\right)=O\left(\sigma_{-\frac{1}{2}}(v)\right) \sum_{j=0}^{\infty} O_{j}(1) s^{j}, \\
x^{s}=\sum_{j=0}^{\infty} O_{j}\left(\log ^{j} x\right) s^{j} .
\end{gathered}
$$

We prove (3.3). Since $H_{v, r}\left(s+\omega^{\prime}\right)$ is holomorphic at the origin, it has a Maclaurin expansion, the coefficient of the $j$ th term of which is

$$
\begin{aligned}
\frac{1}{2 \pi i} \int_{|s|=\frac{1}{4}} H_{v, r}\left(s+\omega^{\prime}\right) s^{-j-1} d s \\
\quad \ll j \prod_{p \mid v}\left(1-p^{\frac{1}{4}-\omega^{\prime}}\right)^{-1} \\
\quad \ll \prod_{p \mid v}\left(1+p^{-\frac{1}{2}}\right) \ll \sigma_{-\frac{1}{2}}(v) .
\end{aligned}
$$

This yields (3.3).

Multiplying the above power series, we obtain

$$
P_{v, r}\left(s+\omega^{\prime}\right) x^{s}=\sigma_{-\frac{1}{2}}(v)\left(O\left(\omega^{\prime}-1\right)+\sum_{j=1}^{\infty} O_{j}\left(\log ^{j-1} x\right)\left(1+\left(\omega^{\prime}-1\right) \log x\right) s^{j}\right) .
$$

Thus, we have

$$
R_{v, r}(x) \ll_{k} \sigma_{-\frac{1}{2}}(v)\left(\log ^{k-1} x\right)\left(1+\left(\omega^{\prime}-1\right) \log x\right) .
$$

Substituting this in the expression for $E_{r}$ and using (3.1) and (3.2) we see that

$$
\begin{aligned}
E_{r} & \ll{ }_{k} \sum_{m<z} m^{\omega-2 \omega^{\prime}} \sum_{a \mid m} a^{-\omega}\left(\sigma_{-\frac{1}{2}}(m) \log ^{k-1} z\right)^{2} \\
& \ll{ }_{k} \log ^{2 k-2} z \sum_{m<z} m^{\omega-2 \omega^{\prime}} \sigma_{-\frac{1}{2}}^{3}(m) \\
& \ll \log ^{2 k-1} z .
\end{aligned}
$$

To complete the proof, we note finally that

$$
\zeta(\omega) \ll(\omega-1)^{-1} \ll \log z .
$$


The next two lemmas are corollaries of lemma 3.

LemMA 4. If $a_{n}$ is defined by (1.8), $\sigma \geqq \frac{1}{2}-y$ and $y \ll\left(\log z_{1}\right)^{-1}$, then

$$
\sum_{z_{1}<n \leqq M} a_{n}^{2} n^{-2 \sigma} \ll M^{O\left(1 / \log z_{1}\right)} z_{1}^{1-2 \sigma}\left(\log \left(z_{2} / z_{1}\right)\right)^{-2}\left(\log z_{2}\right)^{2} .
$$

Graham [1] has given an asymptotic formula for $\sum_{1 \leqq n \leqq M} a_{n}^{2}$, for $M>z_{1}$.

\section{Proof of Lemma 4. Writing}

$$
L_{d}(z)= \begin{cases}\mu(d) \log (z / d), & \text { for } d \leqq z, \\ 0, & \text { for } d>z,\end{cases}
$$

we have

$$
a_{n}=\left(\log \left(z_{2} / z_{1}\right)\right)^{-1} \sum_{d \mid n}\left(L_{d}\left(z_{2}\right)-L_{d}\left(z_{1}\right)\right) .
$$

We note also that, for $z_{1}<n \leqq M$,

$$
n^{-2 \sigma} \ll n^{-1-1 / \log z_{1}} M^{O\left(1 / \log z_{1}\right)} z_{1}^{1-2 \sigma} .
$$

Hence it remains to prove that, for $i=1,2$,

$$
\sum_{z_{1}<n \leqq M}\left(\sum_{d \mid n} L_{d}\left(z_{i}\right)\right)^{2} n^{-1-1 / \log z_{1}} \ll\left(\log z_{2}\right)^{2} .
$$

This follows from lemma 3 with $k=r=1, \omega=\omega^{\prime}$.

Lemma 5. If $b_{n}(x)$ is defined by (1.9), $x \ll\left(\log z_{1}\right)^{-1}$ and

$$
\left\{\begin{array}{l}
\log z_{2} \ll \log v_{1}, \\
\log v_{2} \ll \log z_{1},
\end{array}\right.
$$

then

$$
\sum_{n \leqq v_{2} z_{2}} b_{n}^{2}(x) n^{-1} \ll\left(\log \left(z_{2} / z_{1}\right) \log \left(v_{2} / v_{1}\right)\right)^{-2}\left(\log z_{2}\right)^{4}
$$

Proof. Let

$$
\begin{aligned}
& K_{d}(z)= \begin{cases}\log (z / d), & \text { for } d \leqq z, \\
0, & \text { for } d>z,\end{cases} \\
& L_{d}(z)=\mu(d) K_{d}(z) .
\end{aligned}
$$

Then 


$$
\begin{aligned}
b_{n}(x)=\left(\log \left(z_{2} / z_{1}\right)\right. & \left.\log \left(v_{2} / v_{1}\right)\right)^{-1} \times \\
& \times \sum_{d \mid n}\left(L_{d}\left(z_{2}\right)-L_{d}\left(z_{1}\right)\right)\left(K_{n / d}\left(v_{2}\right)-K_{n / d}\left(v_{1}\right)\right) d^{x} .
\end{aligned}
$$

Thus, we have to show that

$$
\sum_{n \leqq v_{1}, j}\left(\sum_{d \mid n} L_{d}\left(z_{j}\right) K_{n / d}\left(v_{i}\right) d^{x}\right)^{2} n^{-1} \ll\left(\log z_{2}\right)^{4}
$$

for $i, j=1,2$.

For $n \leqq v z$, we have

$$
\begin{aligned}
& \sum_{d \mid n} L_{d}(z) K_{n \mid d}(v) d^{x}= \sum_{\substack{d \mid n \\
n / v<d<z}} \mu(d) \log (z / d) \log (v d / n) d^{x} \\
&= \sum_{\substack{d \mid n \\
d<z}} \mu(d) \log (z / d) \log (v d / n) d^{x} \\
&-\sum_{d \mid n} \mu(d) \log (z / d) \log (v d / n) d^{x}+ \\
&+\sum_{\substack{d \mid n \\
d<v}} \mu(n / d) \log (z d / n) \log (v / d)(n / d)^{x} \\
&= \sum_{r=1}^{7} c_{r}(n), \\
& c_{1}(n)= \sum_{\substack{d \mid n \\
d<z}} \mu(d) \log (z / d) \log (v z / n) d^{x}, \\
& c_{2}(n)=-\sum_{\substack{d \mid n \\
d<z}} \mu(d) \log { }^{2}(z / d) d^{x}, \\
& c_{3}(n)=-\sum_{d \mid n} \mu(d) \log (v z / d) \log \left(v^{3} z / n\right) d^{x}, \\
& c_{4}(n)= \sum_{d \mid n} \mu(d) \log { }^{2}(v z / d) d^{x}, \\
& c_{5}(n)= \sum_{d \mid n} \mu(d) \log v \log \left(v^{2} z / n\right) d^{x}, \\
& c_{6}(n)= n^{x} \sum_{\substack{d \mid n \\
d<v}} \mu(n / d) \log (v / d) \log (v z / n) d^{-x}, \\
&-n^{x} \sum_{d \mid n} \mu(n / d) \log { }^{2}(v / d) d^{-x} \\
& d<v
\end{aligned}
$$


Hence

$$
\sum_{n \leqq v z}\left(\sum_{d \mid n} L_{d}(z) K_{n / d}(v) d^{x}\right)^{2} n^{-1} \ll \sum_{r=1}^{7} S_{r}
$$

where

$$
S_{r}=\sum_{n \leqq v z} c_{r}^{2}(n) n^{-1} .
$$

Consider the sum $S_{5}$. For simplicity, suppose that $x>0$. Denote

$$
f_{n}(x)=n^{-\frac{1}{2}} \sum_{d \mid n} \mu(d)(d / v z)^{x} .
$$

By Schwarz's inequality, we have

$$
f_{n}^{2}(x)=\left(\int_{0}^{x} f_{n}^{\prime}(y) d y\right)^{2} \leqq x \int_{0}^{x}\left(f_{n}^{\prime}(y)\right)^{2} d y,
$$

for $n>1$. Hence, there exists a number $\xi$ such that $0<\xi<x$ and

$$
\sum_{1<n \leqq v z} f_{n}^{2}(x) \leqq x^{2} \sum_{n \leqq v z}\left(f_{n}^{\prime}(\xi)\right)^{2} .
$$

Therefore,

$$
S_{5} \leqq\left(\log v \log \left(v^{2} z\right)\right)^{2}\left(1+(v z)^{2 x} x^{2} S_{5}^{\prime}\right)
$$

where

$$
S_{5}^{\prime}=\sum_{n \leqq v z}\left(\sum_{d \mid n} \mu(d) \log (d / v z) d^{\xi}\right)^{2} n^{-1}
$$

Estimating the sums $S_{r}, r \neq 5$, and $S_{5}^{\prime}$ by Lemma 3, we obtain (3.5). Note that the trivial restriction $d<v z$ may be imposed on those sums, where $d$ runs through all the divisors of $n$.

\section{Mean value estimates.}

In order to be able to treat simultaneously two cases we define the symbol $m_{k}=m_{k, t_{1}, t_{2}}$, for $t_{1}<t_{2}$, as follows:

$$
\begin{gathered}
f(s)=m_{0}(f(s)), \quad \text { for any } t \in\left[t_{1}, t_{2}\right], \\
\int_{t_{1}}^{t_{2}} f(s) d t=m_{1}(f(s)) .
\end{gathered}
$$

The following general lemma is proved in chapter 6 of [4]. 
Lemma 6. Let

$$
K(s, \chi)=\sum_{n=M+1}^{M+N} c_{n} \chi(n) n^{-s},
$$

where $\chi$ is a character $\bmod q$. Then, for $k=0,1$, we have

$$
\sum_{\chi \bmod q} m_{k}\left(|K(i t, \chi)|^{2}\right) \ll\left(q\left(t_{2}-t_{1}\right)^{k}+N\right) \sum_{n=M+1}^{M+N}\left|c_{n}\right|^{2} .
$$

Lemma 7. If $S(s, X, \chi)$ is defined by (2.2), $y \ll 1 / \log z_{1}, \sigma \geqq \frac{1}{2}-y, X>1, h>1$, and $z_{1} \leqq 2 X$, then

$$
\begin{aligned}
\sum_{\chi \bmod q} m_{k}\left(|S(s, X, \chi)|^{2}\right) & \\
& \ll z_{1}^{1-2 \sigma}\left(q\left(t_{2}-t_{1}\right)^{k}+X\right) X^{O\left(1 / \log z_{1}\right)}\left(\log \left(z_{2} / z_{2}\right)\right)^{-2}\left(\log z_{2}\right)^{2},
\end{aligned}
$$

for $k=0,1$.

Proof. Denote by $S_{r}$ the part of the sum $S(s, X, \chi)$ corresponding to the indices $2^{r} X<n \leqq 2^{r+1} X$, for $r \geqq 1$, and by $S_{0}$ the part corresponding to the indices $z_{1}<n \leqq 2 X$. Thus,

$$
S(s, X, \chi)=\sum_{r=0}^{\infty} S_{r} .
$$

By Schwarz's inequality the left hand side of (4.1) is

$$
\ll \sum_{r=0}^{\infty}(r+1)^{2} \sum_{x} m_{k}\left(\left|S_{r}\right|^{2}\right) \text {. }
$$

Here the inner sum is, by Lemma 6 ,

$$
\ll\left(q\left(t_{2}-t_{1}\right)^{k}+2^{r} X\right) \sum_{z_{1}<n \leqq 2^{r+1} X} a_{n}^{2} n^{-2 \sigma} \exp \left(-2^{r h}\right) .
$$

Hence, using Lemma 4 and the fact that $h>1$, we obtain the desired result.

LEMMA 8. If $I_{0}(s, X, \chi)$ is defined by (2.3), $y \ll 1 / \log z_{1}, \sigma>\frac{1}{2}-y, h>2 \sigma, 1<X_{1}$ $<X_{2}$ and (3.4) is valid, then

$$
\sum_{\chi \bmod q} m_{k}\left(\left|\left(\log \left(X_{2} / X_{1}\right)\right)^{-1} \int_{X_{1}}^{X_{2}} X^{-1} I_{0}(s, X, \chi) d X\right|^{2}\right)
$$

$\ll X_{1}^{1-2 \sigma}\left(q\left(t_{2}-t_{1}\right)^{k}+v_{2} z_{2}\right)\left(y \log \left(X_{2} / X_{1}\right) \log \left(z_{2} / z_{1}\right) \log \left(v_{2} / v_{1}\right)\right)^{-2} \times$

$$
\left.\times \log z_{2}\right)^{4}\left(q\left(\max \left(\left|t_{1}\right|,\left|t_{2}\right|\right)+1\right)\right)^{4 y} X_{2}^{2 y},
$$

for $k=0,1$. 
Proof. We have

$$
\int_{X_{1}}^{X_{2}} X^{-1} I_{0}(s, X, \chi) d X=I_{0}^{*}\left(s, X_{2}, \chi\right)-I_{0}^{*}\left(s, X_{1}, \chi\right),
$$

where the asterisque means that $w$ is replaced by $w^{2}$ in the denominator of the integrand. For $w \in C_{0}$, we have

$$
\begin{gathered}
\psi(s+w, \chi) \ll(q(|t|+1))^{2 y}, \\
\Gamma(1+w / h) \ll 1 .
\end{gathered}
$$

The estimate (4.4) follows from Lemma 1. By (4.3)-(4.5) and Schwarz's inequality the square of the left hand side of (4.3) is

$$
\ll(q(|t|+1))^{4 y} X_{1}^{1-2 \sigma} X_{2}^{2 y} y^{-1} \int_{C_{0}}|M(s+w, \chi) F(1-s-w, \bar{\chi})|^{2}\left|w^{-2}\right||d w| .
$$

Hence, in order to obtain (4.2), it remains to prove that, for $w \in C_{0}$,

$$
\begin{gathered}
\sum_{\chi} m_{k}\left(|M(s+w, \chi) F(1-(\overline{s+w}), \chi)|^{2}\right) \\
\ll\left(q\left(t_{2}-t_{1}\right)^{k}+v_{2} z_{2}\right)\left(\log \left(z_{2} / z_{1}\right) \log \left(v_{2} / v_{1}\right)\right)^{-2}\left(\log z_{2}\right)^{4} .
\end{gathered}
$$

For any complex $z$, we have

$$
M(z, \chi) F(1-\bar{z}, \chi)=\sum_{n<v_{2} z_{2}} \chi(n) n^{\bar{z}-1} b_{n}(1-2 \operatorname{Re} z),
$$

where $b_{n}(\cdot)$ is defined by (1.9). Hence, by Lemma 6 , the left hand side of (4.6) is

$$
\ll\left(q\left(t_{2}-t_{1}\right)^{k}+v_{2} z_{2}\right) \sum_{n<v_{2} z_{2}} n^{2 \sigma+2 \operatorname{Re} w-2} b_{n}^{2}(1-2 \sigma-2 \operatorname{Re} w) .
$$

Since $w \in C_{0}, 2 \sigma+2 \operatorname{Re} w-2 \leqq-1$ and $1-2 \sigma-2 \operatorname{Re} w \ll y \ll 1 / \log z_{1}$, so that an application of Lemma 5 yields (4.6).

Lemma 9. If $I_{1}(s, X, \chi)$ is defined by (2.3), $0<y<\frac{1}{4}, \frac{1}{2}-y \leqq \sigma \leqq 2,4<h<A$ and $X>1$, then

$$
I_{1}(s, X, \chi) \ll_{A}\left(q(|t|+1) z_{2} / X v_{1}\right)^{h / 2+\frac{1}{2}-\sigma}\left(v_{1} z_{2}\right)^{\frac{1}{2}} X^{\frac{1}{2}-\sigma} .
$$

Proof. If $w \in C_{1}$, then $\operatorname{Re}(s+w)=\sigma-h / 2$. Hence, for $w \in C_{1}$,

$$
\begin{gathered}
M(s+w, \chi) \ll z_{2}^{h / 2+1-\sigma}, \\
L(1-s-w, \bar{\chi})-F(1-s-w, \bar{\chi}) \ll v_{1}^{\sigma-h / 2} .
\end{gathered}
$$

We have, by Stirling's formula and Lemma 1, 
ON THE ZEROS OF THE DIRICHLET L-FUNCTIONS ...

$$
\begin{aligned}
& \int_{C_{1}}\left|\Psi(s+w, \chi) \Gamma(1+w / h) X^{w} w^{-1}\right||d w| \\
& \ll X^{-h / 2} \int_{-\infty}^{\infty}|\Psi(\sigma-h / 2+i(t+h \omega), \chi)| e^{-\pi|\omega| / 2} d \omega \\
& \ll{ }_{A} X^{-h / 2}(q(|t|+1))^{\frac{1}{2}-\sigma+h / 2} .
\end{aligned}
$$

Combining the previous estimates completes the proof.

\section{Proof of the theorem.}

We state without proof the following slightly modified version of a lemma of Selberg ([8, Lemma 14]).

LEMMA 10. Let $f(s)$ be holomorphic in the rectangle $\sigma_{1} \leqq \sigma \leqq \sigma_{2}, t_{1} \leqq t \leqq t_{2}$, where $\sigma_{2}>\operatorname{Re} \varrho$ whenever $\varrho$ is a zero of $f(s)$ such that $t_{1} \leqq \operatorname{Im} \varrho \leqq t_{2}$. Writing $f(s)$ $=1-g(s)$, suppose that $\left|g\left(\sigma_{2}+i t\right)\right| \leqq \frac{1}{2}$, for $t_{1} \leqq t \leqq t_{2}$. Then we have

$$
\begin{aligned}
\left(t_{2}-t_{1}\right) \sum_{\substack{\rho, f(\varrho)=0 \\
\text { Re } Q>\sigma_{1} \\
t_{1}<\operatorname{Im} \varrho<t_{2}}} \sin \left(\pi \frac{\operatorname{Im} \varrho-t_{1}}{t_{2}-t_{1}}\right) \sinh \left(\pi \frac{\operatorname{Re} \varrho-\sigma_{1}}{t_{2}-t_{1}}\right) \\
\ll \int_{t_{1}}^{t_{2}}\left|g\left(\sigma_{1}+i t\right)\right| d t+\exp \left(\pi \frac{\sigma_{2}-\sigma_{1}}{t_{2}-t_{1}}\right) \int_{t_{1}}^{t_{2}}\left|g\left(\sigma_{2}+i t\right)\right| d t \\
+\int_{\sigma_{1}}^{\sigma_{2}} \exp \left(\pi \frac{\sigma-\sigma_{1}}{t_{2}-t_{1}}\right)\left(\left|g\left(\sigma+i t_{1}\right)\right|+\left|g\left(\sigma+i t_{2}\right)\right|\right) d \sigma .
\end{aligned}
$$

We may suppose that $\alpha \leqq 1$ and $T_{2}-T_{1}>10^{2 / \varepsilon} / \log 2 q$. Let

$$
t_{1}=\left(3 T_{1}-T_{2}\right) / 2, \quad t_{2}=\left(3 T_{2}-T_{1}\right) / 2,
$$

whence

$$
t_{2}-t_{1}=2\left(T_{2}-T_{1}\right)
$$

In particular,

$$
t_{2}-t_{1}>2 \pi / \varepsilon \log 2 q .
$$

Let $T=t_{2}-t_{1}+1$, whence

$$
q T>10^{2 / \varepsilon} .
$$

Let

$$
\begin{aligned}
& \alpha_{0}=\alpha-1 / \log q T, \\
& z_{1}=q^{c / 2-\varepsilon / 2} T^{1 / 2-\varepsilon / 2}, \quad z_{2}=z_{1}(q T)^{\varepsilon / 6} .
\end{aligned}
$$


In particular, by (5.4),

$$
z_{1}>10 \text {. }
$$

Recalling (1.7), let

$$
g(s, \chi)=(M(s, \chi) L(s, \chi)-1)^{2} .
$$

Then, for $k=0,1$ and $\alpha_{0} \leqq \sigma \leqq 2$, we have

$$
\begin{aligned}
& \sum_{\chi \bmod q}^{\prime} m_{k}(|g(s, \chi)|) \\
& \ll_{\varepsilon} q^{1-(c-\varepsilon)\left(\sigma-\frac{1}{2}\right)}(T-k) T^{-(1-\varepsilon)\left(\sigma-\frac{1}{2}\right)} .
\end{aligned}
$$

To see this, we first fix the parameters that occur in lemmas $2,7,8$, and 9 as follows:

$$
\begin{aligned}
v_{1} & =q^{1-c / 2} T^{1 / 2}, \quad v_{2}=v_{1}(q T)^{\varepsilon / 6}, \\
X_{1} & =(q T)^{1-\varepsilon / 6}, \quad X_{2}=q T, \\
y & =1 / \log q T, \\
h & =4+6 / \varepsilon .
\end{aligned}
$$

By Lemma 2 the left hand side of (5.8) is

$$
\ll B_{1}+B_{2}+B_{3}+B_{4},
$$

where

$$
B_{j}=\sum_{x}^{\prime} m_{k}\left(\left|\left(\log \left(X_{2} / X_{1}\right)\right)^{-1} \int_{X_{1}}^{X_{2}} X^{-1} A_{j} d X\right|^{2}\right)
$$

and

$$
\begin{aligned}
& A_{1}=\exp \left(-X^{-h}\right)-1, \\
& A_{2}=S(s, X, \chi), \\
& A_{j}=I_{j-3}(s, X, \chi) ; \quad j=3,4 .
\end{aligned}
$$

Obviously,

$$
B_{1} \ll q\left(t_{2}-t_{1}\right)^{k}(q T)^{-6+4 \varepsilon / 3-12 / \varepsilon} .
$$

By Schwarz's inequality,

$$
B_{2} \ll\left(\log \left(X_{2} / X_{1}\right)\right)^{-1} \sum_{\chi}^{\prime} m_{k}\left(\int_{X_{1}}^{X_{2}} X^{-1}\left|A_{2}\right|^{2} d X\right) .
$$


Hence, by Lemma 7 ,

$$
B_{2} \ll_{\varepsilon} q^{-(c-\varepsilon)\left(\sigma-\frac{1}{2}\right)} T^{-(1-\varepsilon)\left(\sigma-\frac{1}{2}\right)}\left(q\left(t_{2}-t_{1}\right)^{k}+q T / \log q T\right) .
$$

By (1.5) and (5.1),

$$
\max \left(\left|t_{1}\right|,\left|t_{2}\right|\right) \ll q^{1-c} T,
$$

whence, by Lemma 8 ,

$$
B_{3} \ll_{\varepsilon}(q T)^{-(2-\varepsilon / 3)\left(\sigma-\frac{1}{2}\right)}\left(q\left(t_{2}-t_{1}\right)^{k}+(q T)^{1-\varepsilon / 6}\right) .
$$

By Lemma 9,

$$
B_{4} \ll_{\varepsilon} q\left(t_{2}-t_{1}\right)^{k}\left(\frac{q\left(\max \left(\left|t_{1}\right|,\left|t_{2}\right|\right)+1\right) z_{2}}{X_{1} v_{1}}\right)^{h+1-2 \sigma} v_{1} z_{2} X_{1}^{1-2 \sigma},
$$

whence, by (5.9),

$$
B_{4} \ll_{\varepsilon} q\left(t_{2}-t_{1}\right)^{k}(q T)^{-(2-\varepsilon / 3)\left(\sigma-\frac{1}{2}\right)-\varepsilon / 2} .
$$

Combining these estimates gives (5.8).

Recalling (5.7) we define

$$
\Phi(s, \chi)=1-g(s, \chi) .
$$

Obviously each zero of an $L$-function is a zero of the corresponding $\Phi$-function, too. Thus, since $\alpha-\alpha_{0}=1 / \log q T$ and $\frac{1}{4} \leqq\left(t-t_{1}\right) /\left(t_{2}-t_{1}\right) \leqq \frac{3}{4}$, for $T_{1} \leqq t \leqq T_{2}$, we have (see (1.1), (1.3))

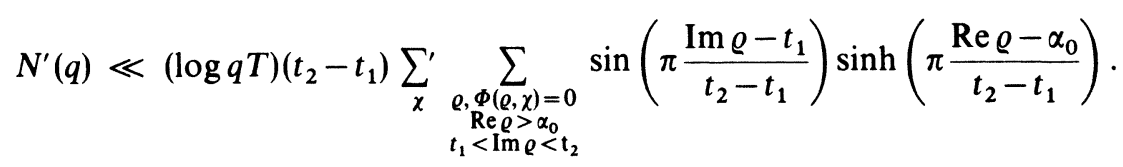

Here we estimate the right hand side by Lemma 10 with $\sigma_{1}=\alpha_{0}$ and $\sigma_{2}=2$. Note that by (5.6), $|g(s, \chi)| \leqq\left(\sum_{n>z_{1}} \tau(n) n^{-2}\right)^{2} \leqq \frac{1}{2}$. Hence, we find

$$
N^{\prime}(q) \ll(U+V) \log q T,
$$

where

$$
U=\sum_{\chi}^{\prime} \int_{t_{1}}^{t_{2}}\left|g\left(\alpha_{0}+i t, \chi\right)\right| d t+q^{\varepsilon} \sum_{\chi}^{\prime} \int_{t_{1}}^{t_{2}}|g(2+i t, \chi)| d t
$$

and

$$
V=\sum_{j=1}^{2} \sum_{\chi}^{\prime} \int_{\alpha_{0}}^{2} q^{\varepsilon\left(\sigma-\alpha_{0}\right) / 2}\left|g\left(\sigma+i t_{j}, \chi\right)\right| d \sigma .
$$

Here we made use of (5.3). 
Now we shall estimate $U$ and $V$ by (5.8). Writing $A(c, \sigma)=(c-\varepsilon)\left(\sigma-\frac{1}{2}\right)$, we have, since $c \geqq 2 \varepsilon$,

$$
U \ll_{\varepsilon} q^{1-A\left(c, \alpha_{0}\right)}\left(t_{2}-t_{1}\right) T^{-A\left(1, \alpha_{0}\right)}
$$

and

$$
\begin{aligned}
V & \ll_{\varepsilon} \int_{\alpha_{0}}^{2} q^{\varepsilon\left(\sigma-\alpha_{0}\right) / 2+1-A(c, \sigma)} T^{1-A(1, \sigma)} d \sigma \\
& \ll_{\varepsilon} q^{1-A\left(c, \alpha_{0}\right)} T^{1-A\left(1, \alpha_{0}\right)} / \log q T .
\end{aligned}
$$

Now the theorem has been proved in the case that $\chi_{0}$ is omitted in the summation, for by (5.5) and (5.2), $\alpha_{0}$ can be replaced by $\alpha$ and the numbers $t_{i}$ by the $T_{i}$ 's.

Finally we note that $N_{\chi_{0}}\left(\alpha ; T_{1}, T_{2}\right)$ is also absorbed in the right hand side of (1.6). In case $T_{2}-T_{1}>4$ this follows from (1.4) and (1.5). In case $4 \geqq T_{2}-T_{1}$ $\gg_{\varepsilon} 1 / \log 2 q$ it follows from (1.5) and the well-known estimate

$$
N_{\chi_{0}}\left(\frac{1}{2} ; Y, Y+1\right) \ll \log (|Y|+2) .
$$

Thus, the proof is complete.

\section{REFERENCES}

1. S. Graham, An asymptotic estimate related to Selberg's sieve, J. Number Theory 10 (1978), 83-94.

2. A. E. Ingham, On the estimation of $N(\sigma, T)$, Quart. J. Math. Oxford Ser.(1) 11 (1940), 291-292.

3. M. Jutila, Zeros of the zeta-function near the critical line, in Studies in pure mathematics, to the memory of Paul Turàn, eds. P. Erdös, L. Alpàr, G. Halàsz and A. Sarközy, Birkhäuser, Basel, 1982.

4. H. L. Montgomery, Topics in multiplicative number theory (Lecture Notes in Math. 227), Springer-Verlag, Berlin - Heidelberg - New York, 1971.

5. Y. Motohashi, Primes in arithmetic progressions, Invent. Math. 44 (1978), 163-178.

6. K. Ramachandra, A simple proof of the mean fourth power estimate for $\zeta\left(\frac{1}{2}+i t\right)$ and $L\left(\frac{1}{2}+i t, \chi\right)$, Ann. Scuola Norm. Sup. Pisa Cl. Sci.(4) 1 (1974), 81-97.

7. A. Selberg, Contributions to the theory of the Riemann zeta-function, Arch. Math. Naturvid. B 48 (1946), No. 5, 89-155.

8. A. Selberg, Contributions to the theory of Dirichlet's L-function, Skr. Norske Vid.-Akad. Oslo I (1946), No. 3, 3-62. 